\title{
Monitoring of 1-22 GHz Instantaneous Spectra of 550 Compact Extragalactic Objects in 1997-1998
}

\author{
Y.Y. Kovalev ${ }^{1}$, N.A. Nizhelsky ${ }^{2}$, Yu.A. Kovalev ${ }^{1}$, V.N. Sidorenkov ${ }^{3}$, \\ M.G. Mingaliev ${ }^{2}$, A.V. Bogdantsov ${ }^{2}$ \\ ${ }^{1}$ Astro Space Center of the Lebedev Physical Institute, \\ Profsoyuznaya 84/32, Moscow, 117810 Russia \\ ${ }^{2}$ Special Astrophysical Observatory, N. Arkhyz, 357147 Russia \\ ${ }^{3}$ Sternberg Astronomical Institute, Universitetskij pr. 13, Moscow, \\ 119899 Russia
}

Abstract. First results of six frequency observations at five epochs in March, 1997, - April, 1998, and a statistical analysis are presented.

\section{Results}

Results presented of 1997-1998 instantaneous spectral observations at 31, 13, $7.6,3.9,2.7, \& 1.4 \mathrm{~cm}$ of a full sample from the Preston et al. (1985) survey with correlated flux at $13 \mathrm{~cm}$ more than $0.1 \mathrm{Jy}$ at declination $-30^{\circ}<\delta<+42^{\circ}$ are part of the long-term monitoring program (details in Kovalev, 1998).

Results are presented on Figure 1 for quasars, BL Lacs, and other objects (radio galaxies, unidentified, etc.). We have constructed the "average" spectra for each subsample using averaged values of spectral indices obtained. All spectra were transfered to the rest frame of the sources before calculations, excluding the sources with unknown redshift value from the analysis. It has been done for the list of "EGRET objects" from Mukherjee et al. (1997) as well. Each of the spectra can be presented as a sum of a spectrum of an extended optically thin component, dominating at $1-4 \mathrm{GHz}$ for all spectra excluding BL Lac's, and a spectrum of a compact component, dominating at frequencies above $4 \mathrm{GHz}$ (similar to the earlier samples, see Kovalev, 1998). The well defined HF turnover for BL Lacs, quasars and EGRET objects is in the range of $12-25 \mathrm{GHz}$. One can see that the BL Lacs average spectrum is the most flat. Only the BL Lacs spectrum is increasing with frequency above $1 \mathrm{GHz}$. This might indicate that for the BL Lacertae objects the relative contribution of the optically thin extended LF component to the total emission is the smallest one. On the contrary, the spectrum of galaxies is dominated by the extended component emission. It can be explained by the orientation and relativistic beaming effects of a compact jet in AGNs. Other explanation can account for the different parent populations.

A contribution of the compact component to the total averaged spectrum is the biggest one for the EGRET sample (excluding complex BL Lacs spectrum). We have also analyzed the speed of variability (like Valtaoja \& Teräsranta, 1996) and have found that the EGRET sources have the greatest at $\lambda>4 \mathrm{~cm}$, next are 
the BL Lacs. This supports the models which involve a connection between HF radio emission (from a compact jet) and $\gamma$ emission (by Compton or synchrotron mechanisms, see e.g. for review von Montigny et al., 1995).

Dagkesamansky (1970) and Gopal-Krishna \& Steppe (1982) pointed out the relation between spectral indices and the flux density for meter-wavelength samples of extragalactic sources. We have found such a correlation as well, but only for the $13 \mathrm{~cm}$ sample of objects - for the subsample of galaxies (see $\mathrm{Ta}$ ble 1). QSOs and BL Lacs do not display such correlation, having a relatively large contribution of the compact component to the spectrum of the total emission (see also Figure 1). This result extends the $\alpha-\lg \left(F_{\nu}\right)$ dependence to the high frequency sample and confirms the previous results obtained from the low frequency ones.

Table 1. A value of a coefficient $k$ (and its error in brackets) for a linear fit like $\alpha=k \lg \left(F_{2.3}\right)+b, F_{\nu} \sim \nu^{\alpha}$.

\begin{tabular}{lccrrr}
\hline $\begin{array}{l}\text { Type of } \\
\text { objects }\end{array}$ & $\begin{array}{c}\text { Number of } \\
\text { objects }\end{array}$ & $\alpha_{2.3-3.9}$ & $\alpha_{3.9-7.7}$ & $\alpha_{7.7-11.1}$ & $\alpha_{11.1-21.7}$ \\
\hline Quasars & 317 & $-0.13(0.07)$ & $-0.15(0.07)$ & $-0.16(0.07)$ & $-0.05(0.06)$ \\
BL Lacs & 56 & $-0.03(0.12)$ & $0.03(0.11)$ & $-0.04(0.11)$ & $-0.28(0.10)$ \\
G & 154 & $-0.57(0.11)$ & $-0.52(0.10)$ & $-0.52(0.11)$ & $-0.47(0.08)$ \\
\hline \hline
\end{tabular}

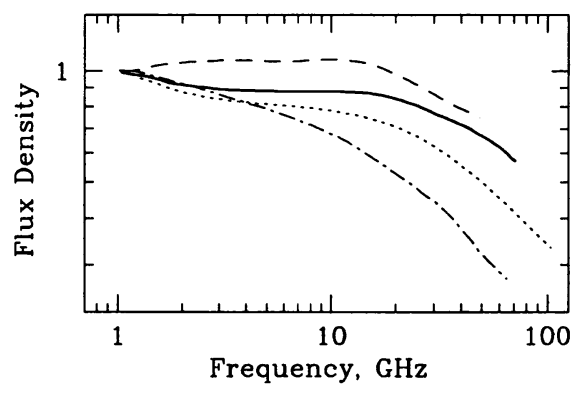

Figure 1. "Averaged" normalized spectra for quasars (a dotted line), for BL Lacertae objects (a dashed line), for other objects (a dot-dashed line), and EGRET objects (a solid line), in the rest frame.

\section{References}

Dagkesamansky, R. D. 1970, Nature, 226, 432

Gopal-Krishna, \& Steppe, H. 1982, A\&A, 113, 150

Kovalev, Yu. A. 1998, Bull. SAO, 44, 50

Preston, R. A., et al. 1985, AJ, 90, 1599

Valtaoja, E., \& Teräsranta, H. 1996, A\&AS, 120, 491

von Montigny, C., et al. 1995, ApJ, 440, 525

Mukherjee, R., et al. 1997, ApJ, 490, 116 